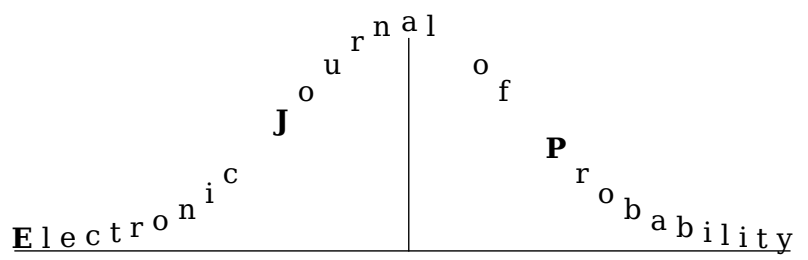

Electron. J. Probab. 25 (2020), article no. 60, 1-17.

ISSN: 1083-6489 https://doi.org/10.1214/20-EJP457

\title{
Restriction of 3D arithmetic Laplace eigenfunctions to a plane
}

\author{
Riccardo W. Maffucci*
}

\begin{abstract}
We consider a random Gaussian ensemble of Laplace eigenfunctions on the 3D torus, and investigate the 1-dimensional Hausdorff measure ('length') of nodal intersections against a smooth 2-dimensional toral sub-manifold ('surface'). A prior result of ours prescribed the expected length, universally proportional to the area of the reference surface, times the wavenumber, independent of the geometry.

In this paper, for surfaces contained in a plane, we give an upper bound for the nodal intersection length variance, depending on the arithmetic properties of the plane. The bound is established via estimates on the number of lattice points in specific regions of the sphere.
\end{abstract}

Keywords: nodal intersections; arithmetic random waves; lattice points on spheres; Gaussian random fields; Kac-Rice formulas.

AMS MSC 2010: $11 \mathrm{P} 21 ; 60 \mathrm{G} 15$.

Submitted to EJP on September 16, 2019, final version accepted on April 12, 2020.

Supersedes arXiv: 1907.09223.

\section{Introduction}

\subsection{Nodal sets for eigenfunctions of the Helmholtz equation}

Let $\Delta_{\mathcal{M}}$ be the Laplace-Beltrami operator, or for short Laplacian, on a smooth manifold $\mathcal{M}$ of dimension $d$. One is interested in eigenfunctions $G$ of the Helmholtz equation

$$
\left(\Delta_{\mathcal{M}}+E\right) G=0
$$

with eigenvalue (or 'energy' in the physics terminology) $E>0$, in the high energy limit $E \rightarrow \infty$. The motivation comes from physics and PDEs [5, 7] - more background and related results may be found in Section 1.7.

Of particular importance is the nodal set (zero-locus) of $G$,

$$
\mathcal{A}_{G}:=\{x \in \mathcal{M}: G(x)=0\} .
$$

${ }^{*}$ EPFL, MA SB Batiment 8, Lausanne, Switzerland.

E-mail: riccardo.maffucci@epfl.ch 
It is known that $\mathcal{A}_{G}$ is a smooth sub-manifold of dimension $d-1$ except for a set of lower dimension [16, Theorem 2.2]. For $d=2$, we call $\mathcal{A}_{G}$ nodal line, and for $d=3$, we call it nodal surface.

Our setting is the three-dimensional standard flat torus $\mathcal{M}=\mathbb{T}^{3}=\mathbb{R}^{3} / \mathbb{Z}^{3}$. Here the Laplace eigenvalues 'energy levels', are of the form $4 \pi^{2} m, m \in S_{3}$, where

$$
S_{3}:=\left\{0<m: m=a_{1}^{2}+a_{2}^{2}+a_{3}^{2}, a_{i} \in \mathbb{Z}\right\} .
$$

The frequencies

$$
\Lambda_{m}=\left\{\lambda \in \mathbb{Z}^{3}:|\lambda|^{2}=m\right\}
$$

are the lattice points on $\sqrt{m} \mathcal{S}^{2}$, the sphere of radius $\sqrt{m}$. The (complex-valued) Laplace eigenfunctions may be written as [8]

$$
G(x)=G_{m}(x)=\sum_{\lambda \in \Lambda} c_{\lambda} e^{2 \pi i\langle\lambda, x\rangle}, \quad x \in \mathbb{T}^{3},
$$

with $c_{\lambda}$ Fourier coefficients.

The eigenspace dimension is the lattice point number, i.e., the number of ways to express $m$ as a sum of three integer squares

$$
N:=|\Lambda|=r_{3}(m) .
$$

In what follows we will always make the (natural) assumption $m \not \equiv 0,4,7(\bmod 8)$, implying ${ }^{1}$

$$
(\sqrt{m})^{1-\epsilon} \ll N \ll(\sqrt{m})^{1+\epsilon}
$$

for all $\epsilon>0[11, \S 1]$ and in particular $N \rightarrow \infty$. This assumption is natural in the sense that if $m \equiv 7(\bmod 8)$ then $m \notin S_{3}$, while multiplying $m$ by 4 just rescales the frequency set [39, §1.3]. Further details on the structure of $\Lambda_{m}$ may be found in Section 3.

\subsection{Nodal intersections}

One insightful approach to the study of the nodal set is given by its restriction to a fixed sub-manifold in the ambient $\mathcal{M}$, the so-called nodal intersections. For $d=2$ unless the curve is contained in the nodal line, the intersection is a set of points. In several situations e.g. $[41,21]$, under appropriate assumptions the nodal intersections number obeys the bound $\ll \sqrt{E}$, where $E>0$ is the eigenvalue.

The nodal set of $G_{m}$ (1.3) is a nodal surface on $\mathbb{T}^{3}$. We consider the restriction of $G_{m}$ to a fixed smooth 2-dimensional sub-manifold $\Pi \subset \mathbb{T}^{3}$, and specifically the nodal intersection length

$$
h_{1}\left(\mathcal{A}_{G} \cap \Pi\right)
$$

where $h_{1}$ is 1-dimensional Hausdorff measure, in the high energy limit $m \rightarrow \infty$. Bourgain and Rudnick found that, for $\Pi$ real-analytic, with nowhere zero Gauss-Kronecker curvature, there exists $m_{\Pi}$ such that for every $m \geq m_{\Pi}$, the surface $\Pi$ is not contained in the nodal set of any eigenfunction $G_{m}$ [8, Theorem 1.2]. Moreover, one has the upper bound

$$
h_{1}\left(\mathcal{A}_{G} \cap \Pi\right)<C_{\Pi} \cdot \sqrt{m}
$$

for some constant $C_{\Pi}\left[9\right.$, Theorem 1.1], and for every eigenfunction $G_{m}$ the nodal intersection is non-empty [9, Theorem 1.3].

\footnotetext{
${ }^{1}$ We will interchangeably use the notations

$$
f=O(g) \quad \text { or } \quad f \ll g
$$

if $|f(k)| \leq c|g(k)|$ for some $c>0$ as $k$ tends to a limit. When $c$ depends on a parameter $t$, we write $f \ll_{t} g$.
} 


\subsection{The arithmetic waves}

The eigenvalue multiplicities allow us to randomise our setting as follows. We will be working with an ensemble of random Gaussian Laplace toral eigenfunctions ('arithmetic waves' for short [34, 37, 24])

$$
F(x)=F_{m}(x)=\frac{1}{\sqrt{N}} \sum_{\lambda \in \Lambda} a_{\lambda} e^{2 \pi i\langle\lambda, x\rangle}, \quad x \in \mathbb{T}^{3},
$$

of eigenvalue $4 \pi^{2} m$, where $a_{\lambda}$ are complex standard Gaussian random variables ${ }^{2}$ (i.e., one has $\mathbb{E}\left[a_{\lambda}\right]=0$ and $\mathbb{E}\left[\left|a_{\lambda}\right|^{2}\right]=1$ ), independent save for the relations $a_{-\lambda}=\overline{a_{\lambda}}$ (so that $F(x)$ is real valued). The total area of the nodal surface of $F$ was studied in $[4,12]$. The arithmetic wave (1.7) may be analogously defined on the $d$-dimensional torus $\mathbb{R}^{d} / \mathbb{Z}^{d}$. Several recent papers investigate the nodal volume $[37,24]$ and nodal intersections of arithmetic waves against a fixed curve [38, 29, 36, 39, 28].

\subsection{Restriction to a surface of nowhere vanishing Gauss-Kronecker curvature}

In [30] we considered the nodal intersection length, i.e. the random variable

$$
\mathcal{L}=\mathcal{L}_{m}:=h_{1}\left(\mathcal{A}_{F_{m}} \cap \Pi\right)
$$

where $\Pi$ is a smooth 2-dimensional sub-manifold of $\mathbb{T}^{3}$, possibly with boundary, admitting a smooth normal vector locally. The expected intersection length is $\mathbb{E}[\mathcal{L}]=\sqrt{m} A \pi / \sqrt{3}$, where $A$ is the total area of $\Pi$ [30, Proposition 1.2]. This expectation is independent of the geometry, and is consistent with (1.6).

The main result of [30] is the precise asymptotic of the nodal intersection length variance, against surfaces of nowhere vanishing Gauss-Kronecker curvature [30, Theorem $1.3]$

where

$$
\operatorname{Var}(\mathcal{L})=\frac{\pi^{2}}{60} \frac{m}{N}\left[3 \mathcal{I}-A^{2}+O\left(m^{-1 / 28+o(1)}\right)\right]
$$

$$
\mathcal{I}=\mathcal{I}_{\Pi}:=\iint_{\Pi^{2}}\left\langle\vec{n}(p), \vec{n}\left(p^{\prime}\right)\right\rangle^{2} d p d p^{\prime}
$$

and $\vec{n}(p)$ is the unit normal vector to $\Pi$ at the point $p$.

In this paper, we consider the other extreme of the nowhere vanishing curvature scenario, namely, the case where $\Pi$ is contained in a plane. The above result for the expected intersection length is valid in this case also. The integral $\mathcal{I}$ satisfies the sharp bounds [30, Proposition 1.4]

$$
\frac{A^{2}}{3} \leq \mathcal{I} \leq A^{2}
$$

so that the leading coefficient of (1.9) is always non-negative and bounded, though it may vanish, for instance when $\Pi$ is a sphere or a hemisphere ${ }^{3}$ : in this case the variance is of lower order than $m / N$. This behaviour is similar to the two-dimensional case, where the analogous leading coefficient (see [38, Theorem 1.2]) is always non-negative and bounded, but may vanish e.g. for circles and semicircles [38, Section 7.2], [36, Section 1.3].

The theoretical maximum of the variance asymptotic is achieved in the case of intersection with a surface contained in a plane. Although excluded by the assumptions of (1.9), this could be approximated arbitrarily well by area- $A$ smooth surfaces with nonvanishing Gauss-Kronecker curvature. We thus expect (1.9) to hold also for $\Pi$ confined to a plane, i.e. we pose the conjecture $\operatorname{Var}(\mathcal{L}) \sim A^{2} m / N \cdot \pi^{2} / 30$ for this case. See [38, Proposition 7.3] for a two-dimensional analogue of this phenomenon.

\footnotetext{
${ }^{2}$ Defined on some probability space $(\Omega, \mathcal{F}, \mathbb{P})$, where $\mathbb{E}$ denotes the expectation with respect to $\mathbb{P}$.

${ }^{3}$ There are also (several) other examples of these so-called 'static' surfaces. To establish the variance asymptotic for these seems to be a difficult problem.
} 


\subsection{Main results}

Let $\Pi$ be a smooth 2-dimensional sub-manifold of $\mathbb{T}^{3}$ contained in a plane. We denote by $\vec{n}=\left(n_{1}, n_{2}, n_{3}\right)$ the unit normal vector to this plane. We distinguish between vectors/planes of the following three types, possibly after relabelling the coordinates and assuming w.l.o.g. that $n_{1} \neq 0$ :

$$
\begin{array}{rll}
n_{2} / n_{1} \in \mathbb{Q} & \text { and } & n_{3} / n_{1} \in \mathbb{Q} ; \\
n_{2} / n_{1} \in \mathbb{Q} & \text { and } & n_{3} / n_{1} \in \mathbb{R} \backslash \mathbb{Q} ; \\
n_{2} / n_{1} \in \mathbb{R} \backslash \mathbb{Q} & \text { and } & n_{3} / n_{1} \in \mathbb{R} \backslash \mathbb{Q} .
\end{array}
$$

Vectors/planes of type (i) will also be called 'rational', and the remaining types 'irrational'. This terminology is borrowed from [28].

As in [9, §2.3] we will denote by $\kappa(R)$ the maximal number of lattice points in the intersection of $R \mathcal{S}^{2}$ and any plane. The upper bound

$$
\kappa(R) \ll R^{\epsilon}, \quad \forall \epsilon>0
$$

is due to Jarnik [23], [9, (2.6)].

Theorem 1.1. Let $\Pi$ be a smooth 2 -dimensional sub-manifold of $\mathbb{T}^{3}$ contained in a plane.

(1) If the plane is rational, then the nodal intersection length variance satisfies the bound

$$
\operatorname{Var}(\mathcal{L}) \ll_{\Pi} \frac{m}{N} \cdot \kappa(\sqrt{m}) .
$$

(2) Moreover, for irrational planes we have

$$
\operatorname{Var}(\mathcal{L}) \ll_{\Pi} \frac{m}{N} \cdot N^{a+\epsilon}
$$

for any positive $\epsilon$ where we may take:

(A) $a=3 / 7$ for planes of type (ii);

(B) $a=3 / 4$ for planes of type (iii).

Theorem 1.1 will be proven in Section 4. Taking into account (1.10), and up to multiplicative constants, the magnitude of the bound $(1.11)$ differs from the conjectured $m / N$ only by a factor of the form $m^{\epsilon}$. Similarly to [38, 39, 30], the above results on expectation and variance have the following consequence.

Theorem 1.2. Let $\Pi$ be a smooth 2 -dimensional sub-manifold of $\mathbb{T}^{3}$ contained in a plane, of total area $A$. Then the nodal intersection length $\mathcal{L}$ satisfies, for all $\epsilon>0$,

$$
\lim _{\substack{m \rightarrow \infty \\ m \neq 0,4,7}} \mathbb{P}\left(\left|\frac{\mathcal{L}}{\sqrt{m}}-\frac{\pi}{\sqrt{3}} A\right|>\epsilon\right)=0 .
$$

Proof. Apply the Chebychev-Markov inequality together with Theorem 1.1 and [30, Proposition 1.2].

Furthermore, one may improve on Theorem 1.1 conditionally on the following conjecture.

Conjecture 1.3 (Bourgain and Rudnick $[9, \S 2.2]$ ). Let $\chi(R, s)$ be the maximal number of lattice points in a cap of radius $s$ of the sphere $R \mathcal{S}^{2}$. Then for all $\epsilon>0$ and $s<R^{1-\delta}$,

$$
\chi(R, s) \ll R^{\epsilon}\left(1+\frac{s^{2}}{R}\right)
$$

as $R \rightarrow \infty$. 
We have the following conditional improvement for planes of type (iii).

Theorem 1.4. Let $\Pi$ be a smooth 2-dimensional sub-manifold of $\mathbb{T}^{3}$ contained in a plane. Assuming Conjecture 1.3, we have that, for every $\epsilon>0$

$$
\operatorname{Var}(\mathcal{L}) \ll_{\Pi} \frac{m}{N} \cdot N^{1 / 2+\epsilon} .
$$

Theorem 1.4 will be proven in Section 4 .

\subsection{Outline of proofs and plan of the paper}

The arithmetic random wave $F(1.7)$ is a random field. For a smooth random field $P: T \subset$ open $\mathbb{R}^{d} \rightarrow \mathbb{R}^{d^{\prime}}$, denote by $\mathcal{V}$ the Hausdorff measure of its nodal set. For instance when $d=3$ and $d^{\prime}=1$ then $\mathcal{V}$ is the nodal area. Only the case $d \geq d^{\prime}$ is interesting, since otherwise the zero set of $P$ is a.s. ${ }^{4}$ empty. Under appropriate assumptions, the moments of $\mathcal{V}$ may be computed via Kac-Rice formulas [2, Theorems 6.8 and 6.9]. These formulas, however, do not apply to our situation [30, Example 1.6] (except in the very special case of the plane containing $\Pi$ being parallel to one of the coordinate planes). To resolve this issue, in [30] we derived Kac-Rice formulas for a random field defined on a surface (also see [25, Theorem 5.3] and [26, Theorems 4.1 and 4.4]), and thus computed $\mathbb{E}[\mathcal{L}]$.

Via an approximate Kac-Rice formula [30, Proposition 1.7], for surfaces of nowhere vanishing Gauss-Kronecker curvature, the problem of computing the nodal intersection length variance (1.9) was reduced to estimating the second moment of the covariance function

$$
r\left(p, p^{\prime}\right):=\mathbb{E}\left[F(p) F\left(p^{\prime}\right)\right]
$$

and of its various first and second order derivatives. The error term in (1.9) comes from bounding the fourth moment of $r$ and of its derivatives.

For $\Pi$ confined to a plane, we wish to prove the upper bounds in Theorem 1.1 by using the following approximate Kac-Rice bound, similarly to [29, 39, 28].

Proposition 1.5 (Approximate Kac-Rice bound). Let $\Pi$ be a smooth 2-dimensional submanifold of $\mathbb{T}^{3}$ contained in a plane. Then we have

$$
\operatorname{Var}(\mathcal{L}) \ll m \iint_{\Pi^{2}}\left(r^{2}+\frac{D \Omega D^{T}}{m}+\frac{\operatorname{tr}(H \Omega H \Omega)}{m^{2}}\right) d p d p^{\prime}
$$

where $D\left(p, p^{\prime}\right), H\left(p, p^{\prime}\right), \Omega$ are appropriate vectors and matrices, depending on $r\left(p, p^{\prime}\right)$, its derivatives, and $\Pi^{5}$.

Proposition 1.5 will be proven in Section 2. The problem of bounding the variance of $\mathcal{L}$ is thus reduced to estimating the second moment of the covariance function $r$ and its various first and second order derivatives. This, in turn, requires estimates for the number of lattice points in specific regions of the sphere $\sqrt{m} \mathcal{S}^{2}$, covered in Section 3.2.

There are marked differences compared to the case of generic surfaces: first, if $\Pi$ is contained in a plane of unit normal $\vec{n}=\left(n_{1}, n_{2}, n_{3}\right)$, it admits everywhere the parametrisation

$$
\begin{aligned}
\gamma: W \subset \mathbb{R}^{2} & \rightarrow \Pi, \\
(u, v) & \mapsto(\zeta+u \xi+v \eta),
\end{aligned}
$$

where $\zeta \in \Pi$ and $\{\vec{n}, \xi, \eta\}$ is an orthonormal basis of $\mathbb{R}^{3}[18, \S 2.5$, Example 1]. The restriction of the wave $F$ to $\Pi$ determines the random field $f: W \subset \mathbb{R}^{2} \rightarrow \mathbb{R}$,

$$
f(u, v):=F(\gamma(u, v))=\frac{1}{\sqrt{N}} \sum_{\lambda \in \Lambda} a_{\lambda} e^{2 \pi i\langle\lambda, \zeta+u \xi+v \eta\rangle} .
$$

\footnotetext{
4The expression 'almost surely', or for short 'a.s.', means 'with probability 1'.

${ }^{5}$ See [30, Definition 3.3].
} 
Then the covariance function (1.14) has the special form

$$
r_{f}\left((u, v),\left(u^{\prime}, v^{\prime}\right)\right)=\frac{1}{N} \sum_{\lambda \in \Lambda} e^{2 \pi i\left\langle\lambda,\left(u^{\prime}-u\right) \xi+\left(v^{\prime}-v\right) \eta\right\rangle},
$$

depending on the difference $\left(u^{\prime}, v^{\prime}\right)-(u, v)$ only: the field $f$ is stationary ${ }^{6}$. This behaviour is very different from the case of generic surfaces. In particular it eventually leads to a different method from [30] of controlling the second moment, and specifically the off-diagonal terms. Indeed, in our previous paper, the off-diagonal terms are handled via a generalisation of Van der Corput's lemma to higher dimensions [30, Proposition 5.4], applicable for surfaces $\Pi$ of nowhere vanishing Gauss-Kronecker curvature. On the other hand if $\Pi$ is confined to a plane, the special form (1.17) of the covariance function allows us to establish the estimates of Lemma 4.5 directly, leading to a different arithmetic problem from the generic surfaces case.

Similarly to $[29,28]$ (nodal intersections against a straight line in two and three dimensions), in the linear case the variance upper bounds depend on the arithmetic properties of the line/plane. In Theorem 1.1, the upper bound is stronger in the case of rational planes, and the bound for planes of type (ii) is stronger than for those of type (iii), again similar to $[29,28]$. This situation occurs because the bounds rely on estimates for lattice points in specific regions of the sphere: when

$$
\frac{n_{3}}{n_{1}}, \frac{n_{3}}{n_{2}}
$$

are irrational numbers, the lattice point estimates are derived using simultaneous Diophantine approximation, so that the bound for the variance is stronger when the number of irrationals to approximate is smaller [28, §8].

\subsection{Connections with other results}

Over the last decade or so, there has been a growing literature on geometric functionals of random fields, and on the asymptotic behaviour of high energy eigenfunctions. The study of the nodal set (1.1) dates back to Hooke's and Chladni's pioneering work (17th-18th century).

The aforementioned Kac-Rice formulas have been used [2] for a long time in various scientific contexts, including telecommunications [35], oceanography [27, 2, 1], and photography [40]. These formulas allow us, under certain assumptions, to compute moments of local functionals for random fields (e.g. measure of zero set, critical points, excursion sets, ... ). Employing the recently introduced fourth-moments method [32] alongside Kac-Rice machinery has allowed to investigate the finer asymptotic behaviour of functionals, such as limiting distribution [31].

The present paper and those cited in Section 1.3 focus on the nodal set of the toral random wave (defined by (1.7) in the three-dimensional case), of interest also to number theorists for its fine arithmetic properties. The case of the sphere and ensemble of random spherical harmonics has also been widely studied, e.g. in [42, 43, 13]. For limiting theorems in the context of random waves on generic manifolds, see e.g. $[14,15,21,41]$.

Berry [6] conjectured that at microscopic scales the toral and spherical ensembles converge to random plane waves (the so-called Berry random wave model RWM). For recent limiting results on the nodal sets for the RWM see e.g. [33] and [17] (threedimensional setting). In another recent development, Beliaev and the author showed that it is possible to couple arithmetic waves so that they converge locally uniformly to the random plane wave and estimated the rate of convergence [3].

\footnotetext{
${ }^{6}$ In particular we may assume w.l.o.g. that $\zeta$ is the origin.
} 


\section{Kac-Rice bound: Proof of Proposition 1.5}

\subsection{Setup}

We fix a smooth 2-dimensional sub-manifold $\Pi$ of $\mathrm{T}^{3}$ confined to a plane, denoting the unit normal $\vec{n}=\left(n_{1}, n_{2}, n_{3}\right)$. Then w.l.o.g. $\Pi$ admits everywhere the parametrisation (cf. (1.16))

$$
\begin{aligned}
\gamma:[0, A] \times[0, B] & \subset \mathbb{R}^{2} \rightarrow \Pi, \\
(u, v) & \mapsto p=u \xi+v \eta,
\end{aligned}
$$

where $\{\vec{n}, \xi, \eta\}$ is an orthonormal basis of $\mathbb{R}^{3}$,

$$
A:=\max \{u: u \xi+v \eta \in \Pi\}, \quad \text { and } \quad B:=\max \{v: u \xi+v \eta \in \Pi\} .
$$

Later we will choose (assuming w.l.o.g that $n_{1} \neq 0$ )

$$
\xi=\frac{\left(n_{2},-n_{1}, 0\right)}{\sqrt{n_{1}^{2}+n_{2}^{2}}}, \quad \eta=\frac{\left(n_{1} n_{3}, n_{2} n_{3},-n_{1}^{2}-n_{2}^{2}\right)}{\sqrt{n_{1}^{2}+n_{2}^{2}}} .
$$

We now introduce some necessary notation for the derivatives of the covariance function $r$ (1.14).

Definition 2.1. Define the row vector $D:=\nabla r$,

$$
D\left(p, p^{\prime}\right)=\frac{2 \pi i}{N} \sum_{\lambda \in \Lambda} e^{2 \pi i\left\langle\lambda, p^{\prime}-p\right\rangle} \cdot \lambda
$$

and the Hessian matrix $H:=\operatorname{Hess}(r)$,

$$
H\left(p, p^{\prime}\right)=-\frac{4 \pi^{2}}{N} \sum_{\lambda \in \Lambda} e^{2 \pi i\left\langle\lambda, p^{\prime}-p\right\rangle} \cdot \lambda^{T} \lambda .
$$

We also introduce the matrix

$$
\Omega:=\left(\begin{array}{ccc}
n_{2}^{2}+n_{3}^{2} & -n_{1} n_{2} & -n_{1} n_{3} \\
-n_{1} n_{2} & n_{1}^{2}+n_{3}^{2} & -n_{2} n_{3} \\
-n_{1} n_{3} & -n_{2} n_{3} & n_{1}^{2}+n_{2}^{2}
\end{array}\right)
$$

\subsection{Proof of Proposition 1.5}

We bring some modifications to the proof of [30, Proposition 1.7]. With the notation of the parametrisation (2.1), consider the rectangle $U$ of vertices the origin, $A \xi, B \eta$, and $A \xi+B \eta$. We partition such a rectangle (with boundary overlaps) into small squares $U_{j}$ of side length $\delta=c_{0} / \sqrt{m}$ for some small $c_{0}>0$. Writing $\Pi_{j}:=\Pi \cap U_{j}$, we define

$$
\mathcal{L}_{j}:=h_{1}\left(\mathcal{A}_{F} \cap \Pi_{j}\right)
$$

recalling the notations $\mathcal{A}_{F}$ (1.1) for the nodal set and $h_{1}$ for Hausdorff measure. Then for (1.8) one has a.s.

$$
\mathcal{L}=\sum_{j} \mathcal{L}_{j}
$$

It follows that

$$
\operatorname{Var}(\mathcal{L})=\sum_{i, j} \operatorname{Cov}\left(\mathcal{L}_{i}, \mathcal{L}_{j}\right)
$$

The set $\Pi^{2}$ is thus partitioned (with boundary overlaps) into regions $\Pi_{i} \times \Pi_{j}=: V_{i, j}$. We call the region $V_{i, j}$ singular if there are points $p \in \Pi_{i}$ and $p^{\prime} \in \Pi_{j}$ s.t. $\left|r\left(p, p^{\prime}\right)\right|>1 / 2$. 
The union of all singular regions is the singular set $S$. It was proven in [30, Lemma 3.12] that

$$
\operatorname{meas}(S) \ll \iint_{\Pi^{2}} r^{2}\left(p, p^{\prime}\right) d p d p^{\prime} .
$$

We partition the summation (2.4) over singular and non-singular regions:

$$
\operatorname{Var}(\mathcal{L})=\sum_{V_{i, j} \text { non-sing }} \operatorname{Cov}\left(\mathcal{L}_{i}, \mathcal{L}_{j}\right)+\sum_{V_{i, j} \text { sing }} \operatorname{Cov}\left(\mathcal{L}_{i}, \mathcal{L}_{j}\right) .
$$

In $[30, \S 3.4]$ we showed the uniform bound

$$
\operatorname{Cov}\left(\mathcal{L}_{i}, \mathcal{L}_{j}\right) \ll \frac{1}{m}
$$

hence

$$
\left|\sum_{V_{i, j} \text { sing }} \operatorname{Cov}\left(\mathcal{L}_{i}, \mathcal{L}_{j}\right)\right| \ll m \iint_{\Pi^{2}} r^{2}\left(p, p^{\prime}\right) d p d p^{\prime}
$$

via (2.5).

For non-singular regions, Kac-Rice formulae yield (see [30, (3.19), §5.2, and §5.3])

$$
\operatorname{Cov}\left(\mathcal{L}_{i}, \mathcal{L}_{j}\right) \ll m \iint_{V_{i, j}}\left(r^{2}+\frac{D \Omega D^{T}}{m}+\frac{t r(H \Omega H \Omega)}{m^{2}}\right) d p d p^{\prime}
$$

with $D, H, \Omega$ as in Definition 2.1. We substitute (2.8) and (2.7) into (2.6), and extend the domain of integration to the whole of $\Pi^{2}$ via another application of (2.5). The proof of Proposition 1.5 is thus complete.

\section{Lattice points on spheres}

\subsection{Background}

To estimate the second moment of the covariance function $r$ and of its derivatives (the RHS of (1.15)), we will need several considerations on lattice points on spheres $\sqrt{m} \mathcal{S}^{2}$. As is well-known, an integer $m$ is representable as a sum of three squares if and only if it is not of the form $4^{l}(8 k+7)$, for $k, l$ non-negative integers. Recall the notation (1.4) $N:=|\Lambda|=r_{3}(m)$ for the number of such representations.

Subtle questions about the distribution of $\Lambda / \sqrt{m}$ in the unit sphere as $m \rightarrow \infty$ are of independent interest in number theory. The limiting equidistribution of the lattice points was conjectured and proved conditionally by Linnik, and subsequently proven unconditionally $[19,20,22]$. The finer statistics of $\Lambda / \sqrt{m}$ on shrinking sets has been recently investigated by Bourgain-Rudnick-Sarnak [11, 10].

Proposition 3.1 ([10, Theorem 1.1]). Fix $0<s<2$. Suppose $m \rightarrow \infty, m \not \equiv 0,4,7$ $(\bmod 8)$. There is some $\delta>0$ so that

$$
\sum_{\lambda \neq \lambda^{\prime}} \frac{m^{s / 2}}{\left|\lambda-\lambda^{\prime}\right|^{s}}=\frac{2^{1-s}}{2-s} \cdot N^{2}+O\left(N^{2-\delta}\right) .
$$

\subsection{Lattice points in spherical caps and segments}

In the present subsection, we collect several bounds for lattice points in certain regions of the sphere. For a more detailed account, see e.g. [9, §2] (spherical caps) and $[28, \S \S 5,6,8]$ (spherical segments).

Definition 3.2 ([28, Definition 4.1]). Given a sphere $\mathfrak{S}$ in $\mathbb{R}^{3}$ with centre $O$ and radius $R$, and a point $P \in \mathfrak{S}$, we define the spherical cap $\mathcal{T}$ to be the intersection of $\mathfrak{S}$ with the 
ball $\mathcal{B}_{s}(P)$ of radius $s$ centred at $P$. We will call $s$ the radius of the cap, and the unit vector $\alpha:=\overrightarrow{O P} / R$ the direction of $\mathcal{T}$.

The intersection of $\mathfrak{S}$ with the boundary of $\mathcal{B}_{s}(P)$ is a circle, called the base of $\mathcal{T}$, and the radius of the base will be denoted by $k$. Let $Q, Q^{\prime}$ be two points on the base which are diametrically opposite (note $\overline{P Q}=\overline{P Q^{\prime}}=s$ ): we define the opening angle of $\mathcal{T}$ to be $\theta=\widehat{Q O Q^{\prime}}$. The height $h$ of $\mathcal{T}$ is the distance between the point $P$ and the disc base.

We will be considering the sphere of radius

$$
R=\sqrt{m}
$$

If $s, h, k$ and $\theta$ denote the radius, height, radius of the base, and opening angle of $\mathcal{T}$ respectively, then geometric considerations give us the relations $0 \leq s \leq 2 R, 0 \leq h \leq 2 R$, $0 \leq k \leq R, 0 \leq \theta \leq \pi$, and

$$
s^{2}=2 R h
$$

Let us introduce the notation

$$
\chi(R, s)=\max _{\mathcal{T}} \#\left\{\lambda \in \mathbb{Z}^{3} \cap \mathcal{T}\right\}
$$

for the maximal number of lattice points contained in any spherical cap $\mathcal{T} \subset R \mathcal{S}^{2}$ of radius $s$.

Lemma 3.3 (Bourgain and Rudnick [9, Lemma 2.1]). We have for all $\epsilon>0$,

$$
\chi(R, s) \ll R^{\epsilon}\left(1+\frac{s^{2}}{R^{1 / 2}}\right)
$$

as $R \rightarrow \infty$.

Compare Lemma 3.3 with Conjecture 1.3. We now introduce another particular region of the sphere, the segment (sometimes called 'slab' or 'annulus').

Definition 3.4. Given a sphere $\mathfrak{S}$ in $\mathbb{R}^{3}$ with centre $O$ and radius $R$, and two parallel planes $\Pi_{1}, \Pi_{2}$, we call spherical segment $\Gamma$ the region of the sphere delimited by $\Pi_{1}, \Pi_{2}$. The two bases of $\Gamma$ are the circles $\mathfrak{S} \cap \Pi_{1}$ and $\mathfrak{S} \cap \Pi_{2}$ : we always assume the latter to be the larger. We define the height $h$ of the spherical segment to be the distance between $\Pi_{1}$ and $\Pi_{2}$. We will denote by $k$ the radius of the larger base.

Moreover, let $\mathfrak{C}$ be a great circle of the sphere $\mathfrak{S}$, lying on a plane perpendicular to $\Pi_{1}$ and $\Pi_{2}$. Denote $\{A, B\}:=\mathfrak{S} \cap \Pi_{1} \cap \mathfrak{C}$ and $\{C, D\}:=\mathfrak{S} \cap \Pi_{2} \cap \mathfrak{C}$. We define the opening angle of $\mathfrak{S}$ to be $\theta=\widehat{A O C}+\widehat{B O D}=2 \cdot \widehat{A O C}$. The direction of the spherical segment is the unit vector $\alpha$ that is the direction of the two spherical caps $\mathcal{T}_{1}, \mathcal{T}_{2}$ satisfying $\mathfrak{S}=\mathcal{T}_{2} \backslash \mathcal{T}_{1}$.

A cap is thus a special case of a segment. It will be convenient to always assume a spherical segment $\Gamma$ to be contained in a hemisphere, so that any two of $h, k, \theta$ completely determine $\Gamma$. We always have $0 \leq h \leq R, 0 \leq k \leq R, 0 \leq \theta \leq \pi$ and the relation [28, Lemma 5.3]

$$
k \theta \ll h
$$

as $R \rightarrow \infty$.

Next, we state two lemmas of [28] which will be needed later.

Lemma 3.5 ([28, Lemma 9.1]). Given $0<c<R$, fix a point $P \in R \mathcal{S}^{2}$, and let $\alpha$ be a unit vector. Then all points $P^{\prime} \in R \mathcal{S}^{2}$ satisfying $\left|\left\langle P-P^{\prime}, \alpha\right\rangle\right| \leq c$ lie on the same spherical segment, of height (at most) $2 c$ and direction $\alpha$ on $R \mathcal{S}^{2}$. 
Lemma 3.6 ([28, Lemma 7.1]). Let $c=c(R)>0$, with $c \rightarrow 0$ as $R \rightarrow \infty$. Fix a point $P \in R \mathcal{S}^{2}$, and let $\alpha$ be a unit vector. Then all points $P^{\prime} \in R \mathcal{S}^{2}$ satisfying $\left|\left\langle P-P^{\prime}, \alpha\right\rangle\right| \leq$ $c\left|P-P^{\prime}\right|$ lie: either on the same spherical segment, of opening angle $8 c+O\left(c^{3}\right)$ and direction $\alpha$; or on the same spherical cap, of radius $\ll c R$ and direction $\alpha$, on $R \mathcal{S}^{2}$.

In [28] we found several upper bounds for the maximal number of lattice points belonging to a spherical segment $\Gamma$ of the sphere $R \mathcal{S}^{2}$,

$$
\psi=\psi(R, h, k, \theta):=\max _{\Gamma} \#\left\{\lambda \in \mathbb{Z}^{3} \cap \Gamma\right\},
$$

with $h, k, \theta$ as in Definition 3.4. Here we collect some of these bounds for convenience. Recall that $\kappa$ denotes the maximal number of spherical lattice points in a plane, and the types (i), (ii), (iii) of vectors/planes defined in Section 1.5.

Proposition 3.7. Let $\Gamma \subset R \mathcal{S}^{2}$ be a spherical segment of opening angle $\theta$, height $h$, radius of larger base $k$, and direction $\alpha$. Then the number of lattice points lying on $\Gamma$ satisfies for every $\epsilon>0$ :

(1) if $\alpha$ is of type (i),

$$
\psi \ll_{\alpha} R^{\epsilon} \cdot(1+h) ;
$$

(2) if $\alpha$ is of type (ii) or (iii),

$$
\psi \ll{ }_{\alpha} R^{1 / 2+\epsilon} \cdot\left(R^{1 / 4}+h\right) ;
$$

(3) if $\alpha$ is of type (ii),

$$
\psi \ll_{\alpha} \kappa(R)\left(1+R \cdot \theta^{1 / 2}\right)
$$

(4) if $\alpha$ is of type (iii),

$$
\psi \ll_{\alpha} \kappa(R)\left(1+R \cdot \theta^{1 / 3}\right) .
$$

Proof. The bound (3.5) was proven in [28, Proposition 6.3] (also see Yesha [44, Lemma A.1]). We now show that (3.6) follows directly from [28]. Applying [28, Proposition 5.4] with $\Omega=R^{1 / 4}$,

so that, by Lemma 3.3,

$$
\psi \ll \chi\left(R, R^{1 / 4}\right) \cdot\left\lceil\frac{k}{R^{1 / 4}}\right\rceil \cdot\left\lceil R^{3 / 4} \theta\right\rceil
$$

$$
\psi \ll R^{\epsilon} \cdot\left(1+\frac{k}{R^{1 / 4}}+R^{3 / 4} \theta+R^{1 / 2} k \theta\right) .
$$

Since $0 \leq k \leq R, 0 \leq \theta \leq \pi$ and $k \theta \ll h$ (3.3), we obtain (3.6). The bounds (3.7) and (3.8) were shown in [28, Proposition 8.3] and [28, Proposition 6.2] respectively.

\section{Proofs of Theorems 1.1 and 1.4}

\subsection{The bounds for the variance}

In this section, we prove Theorem 1.1. We commence by further reducing our problem of bounding the variance to estimating a summation over the lattice points on the sphere. Recall the notations $\Lambda$ of the frequency set (1.2), $A, B \in \mathbb{R}^{+}$(2.2), and vectors/matrices $D, H, \Omega$ (Definition 2.1).

Lemma 4.1. Let $\Pi$ be a 2-dimensional toral sub-manifold confined to a plane. Then

$$
\iint_{\Pi^{2}}\left(r^{2}+\frac{D \Omega D^{T}}{m}+\frac{\operatorname{tr}(H \Omega H \Omega)}{m^{2}}\right) d p d p^{\prime} \ll_{\Pi} \frac{\mathcal{G}}{N^{2}},
$$

where

$$
\mathcal{G}=\mathcal{G}_{m, \Pi}:=\sum_{\lambda, \lambda^{\prime} \in \Lambda_{m}}\left|\int_{0}^{A} \int_{0}^{B} e^{2 \pi i\left\langle\lambda-\lambda^{\prime}, u \xi+v \eta\right\rangle} d u d v\right|^{2} .
$$




\section{Restriction of Laplace eigenfunctions}

The proof of Lemma 4.1 is relegated to appendix A. Assuming it, we deduce the following bound for the nodal intersection length variance.

Corollary 4.2. Let $\Pi$ be a 2-dimensional toral sub-manifold confined to a plane. Then

$$
\operatorname{Var}(\mathcal{L}) \ll_{\Pi} \frac{m}{N^{2}} \cdot \mathcal{G} .
$$

Proof. One substitutes the estimate (4.1) into the approximate Kac-Rice bound (1.15).

In the following two lemmas we bound $\mathcal{G}$, thereby completing the proof of Theorem 1.1. Recall that we distinguish between planes of three types, according to the rationality of the ratios between coordinates of the associated normal vector. Recall further that $\kappa$ denotes the maximal number of spherical lattice points lying on a plane.

Lemma 4.3. Let $\Pi$ be a 2-dimensional toral sub-manifold confined to a rational plane. Then we have

$$
\mathcal{G} \ll_{\Pi} N \cdot \kappa(\sqrt{m}) .
$$

Lemma 4.3 will be proven in Section 4.2. For irrational planes, we have the following. Lemma 4.4. For every $\epsilon>0$, one has

$$
\mathcal{G} \ll_{\Pi} N^{1+a+\epsilon}
$$

where we may take:

(A) $a=3 / 7$ if $\vec{n}$ is of type (ii);

(B) $a=3 / 4$ if $\vec{n}$ is of type (iii);

(C) $a=1 / 2$ conditionally on Conjecture 1.3.

Lemma 4.4 will be proven in Sections 4.3 and 4.4. Assuming them we may complete the proofs of our main theorems.

Proof of Theorems 1.1 and 1.4 assuming Lemmas 4.3 and 4.4. One substitutes (4.4) into (4.3) to obtain (1.11). One substitutes (4.5) into (4.3) to obtain (1.12) and (1.13).

\subsection{Rational planes}

In this subsection we prove Lemma 4.3. We will need a preparatory result, the proof of which will follow in appendix A.

Lemma 4.5. For $\lambda, \lambda^{\prime} \in \Lambda_{m}$ one has

$$
\left|\int_{0}^{A} \int_{0}^{B} e^{2 \pi i\left\langle\lambda-\lambda^{\prime}, u \xi+v \eta\right\rangle} d u d v\right|^{2} \ll_{\Pi} 1 .
$$

Moreover if $\lambda, \lambda^{\prime}$ satisfy

$$
\left\langle\lambda-\lambda^{\prime}, \xi\right\rangle \cdot\left\langle\lambda-\lambda^{\prime}, \eta\right\rangle \neq 0
$$

then

$$
\left|\int_{0}^{A} \int_{0}^{B} e^{2 \pi i\left\langle\lambda-\lambda^{\prime}, u \xi+v \eta\right\rangle} d u d v\right|^{2} \ll \Pi \frac{1}{\left\langle\lambda-\lambda^{\prime}, \xi\right\rangle^{2}\left\langle\lambda-\lambda^{\prime}, \eta\right\rangle^{2}} .
$$

Proof of Lemma 4.3 assuming Lemma 4.5. We split the summation

$$
\mathcal{G}=\sum_{\lambda, \lambda^{\prime}}\left|\int_{0}^{A} \int_{0}^{B} e^{2 \pi i u\left\langle\lambda-\lambda^{\prime}, \xi\right\rangle} d u \cdot e^{2 \pi i v\left\langle\lambda-\lambda^{\prime}, \eta\right\rangle} d v\right|^{2}
$$




\section{Restriction of Laplace eigenfunctions}

over the set of pairs $\left(\lambda, \lambda^{\prime}\right)$ s.t. $\left\langle\lambda-\lambda^{\prime}, \xi\right\rangle \cdot\left\langle\lambda-\lambda^{\prime}, \eta\right\rangle \neq 0$ and its complement. Thanks to Lemma 4.5,

$$
\begin{aligned}
\mathcal{G} & \ll_{\Pi} \#\left\{\left(\lambda, \lambda^{\prime}\right):\left|\left\langle\lambda-\lambda^{\prime}, \xi\right\rangle\right|=0 \vee\left|\left\langle\lambda-\lambda^{\prime}, \eta\right\rangle\right|=0\right\} \\
& +\sum_{\left\langle\lambda-\lambda^{\prime}, \xi\right\rangle \cdot\left\langle\lambda-\lambda^{\prime}, \eta\right\rangle \neq 0} \frac{1}{\left\langle\lambda-\lambda^{\prime}, \xi\right\rangle^{2}\left\langle\lambda-\lambda^{\prime}, \eta\right\rangle^{2}} .
\end{aligned}
$$

We claim that there are few pairs $\left(\lambda, \lambda^{\prime}\right)$ satisfying $\left\langle\lambda-\lambda^{\prime}, \xi\right\rangle=0$. Indeed, once we fix $\lambda$, the lattice point $\lambda^{\prime}$ is confined to the plane

$$
\langle\xi,(x, y, z)\rangle=l,
$$

where $l:=\langle\lambda, \xi\rangle \in \mathbb{R}$. By definition of $\kappa$, there are at most $\kappa(\sqrt{m})$ solutions $(x, y, z) \in \Lambda$ to (4.9). Therefore,

$$
\#\left\{\left(\lambda, \lambda^{\prime}\right):\left|\left\langle\lambda-\lambda^{\prime}, \xi\right\rangle\right|=0\right\}=\sum_{\lambda \in \Lambda} \#\left\{\lambda^{\prime}:\left\langle\lambda^{\prime}, \xi\right\rangle=\langle\lambda, \xi\rangle\right\} \leq N \cdot \kappa(\sqrt{m}) .
$$

Similarly, there are few pairs $\left(\lambda, \lambda^{\prime}\right)$ such that $\left\langle\lambda-\lambda^{\prime}, \eta\right\rangle=0$.

We turn to bounding the summation in (4.8). By assumption, $\vec{n}$ is of type (i). Taking $\xi, \eta$ as in (2.3), then $\xi, \eta$ are also of type (i), hence we may write $\xi=c \tilde{\xi}$ and $\eta=c^{\prime} \tilde{\eta}$, where $\tilde{\xi}, \tilde{\eta} \in \mathbb{Z}^{3}$ and $c, c^{\prime}$ are real numbers. Therefore,

$$
\begin{aligned}
\sum_{\left\langle\lambda-\lambda^{\prime}, \xi\right\rangle \cdot\left\langle\lambda-\lambda^{\prime}, \eta\right\rangle \neq 0} \frac{1}{\left\langle\lambda-\lambda^{\prime}, \xi\right\rangle^{2}\left\langle\lambda-\lambda^{\prime}, \eta\right\rangle^{2}} \\
\ll \Pi \sum_{\lambda} \sum_{a \neq 0} \sum_{b \neq 0} \frac{1}{a^{2}} \frac{1}{b^{2}} \cdot \#\left\{\lambda^{\prime}:\left\langle\tilde{\xi}, \lambda^{\prime}\right\rangle=a \in \mathbb{Z} \wedge\left\langle\tilde{\eta}, \lambda^{\prime}\right\rangle=b \in \mathbb{Z}\right\} .
\end{aligned}
$$

For fixed $a, b$, the lattice point $\lambda^{\prime}$ is confined to the intersection of the two planes

$$
\left\langle\tilde{\xi}, \lambda^{\prime}\right\rangle=a \quad \text { and } \quad\left\langle\tilde{\eta}, \lambda^{\prime}\right\rangle=b .
$$

Since $\tilde{\xi} \perp \tilde{\eta}$, these two planes intersect in a line, hence the number of solutions $\lambda^{\prime} \in \Lambda$ cannot exceed two. It follows that

$$
\sum_{\left\langle\lambda-\lambda^{\prime}, \xi\right\rangle \cdot\left\langle\lambda-\lambda^{\prime}, \eta\right\rangle \neq 0} \frac{1}{\left\langle\lambda-\lambda^{\prime}, \xi\right\rangle^{2}\left\langle\lambda-\lambda^{\prime}, \eta\right\rangle^{2}} \ll N .
$$

Substituting (4.10) and (4.11) into (4.8) yields (4.4).

\subsection{Irrational planes}

In the present subsection we prove Lemma 4.4 parts (A) and (B), using the bounds for lattice points in spherical caps and segments of Section 3.2. We introduce the parameters $c=c(N), \rho=\rho(N)>0$ and consider the three subsets

- first subset: $\left|\left\langle\lambda-\lambda^{\prime}, \xi\right\rangle\right| \leq c$;

- second subset: $\left|\left\langle\lambda-\lambda^{\prime}, \eta\right\rangle\right| \leq \rho\left|\lambda-\lambda^{\prime}\right|$;

- third subset: $\left|\left\langle\lambda-\lambda^{\prime}, \xi\right\rangle\right|>c,\left|\left\langle\lambda-\lambda^{\prime}, \eta\right\rangle\right|>\rho\left|\lambda-\lambda^{\prime}\right|$.

We apply Lemma 4.5 to obtain

$$
\begin{aligned}
& \mathcal{G} \ll_{\Pi} \#\left\{\left(\lambda, \lambda^{\prime}\right):\left|\left\langle\lambda-\lambda^{\prime}, \xi\right\rangle\right| \leq c\right\}+\#\left\{\left(\lambda, \lambda^{\prime}\right):\left|\left\langle\lambda-\lambda^{\prime}, \eta\right\rangle\right| \leq \rho\left|\lambda-\lambda^{\prime}\right|\right\} \\
& +\sum_{\substack{\left|\left\langle\lambda-\lambda^{\prime}, \xi\right\rangle\right|>c \\
\left|\left\langle\lambda-\lambda^{\prime}, \eta\right\rangle\right|>\rho\left|\lambda-\lambda^{\prime}\right|}} \frac{1}{\left\langle\lambda-\lambda^{\prime}, \xi\right\rangle^{2}\left\langle\lambda-\lambda^{\prime}, \eta\right\rangle^{2}} .
\end{aligned}
$$




\section{Restriction of Laplace eigenfunctions}

(A) Let $\vec{n}$ be of type (ii). Taking $\xi, \eta$ as in (2.3), then $\xi$ is of type (i) and $\eta$ of type (ii).

First subset. Once we fix $\lambda$, the lattice points $\lambda^{\prime}$ satisfying

$$
\left|\left\langle\lambda-\lambda^{\prime}, \xi\right\rangle\right| \leq c
$$

lie on a spherical segment $\Gamma_{\lambda}$ of height at most $2 c$ and direction $\xi$ (see Lemma 3.5). As $\xi$ is of type (i), we may apply (3.5):

$$
\#\left\{\left(\lambda, \lambda^{\prime}\right):\left|\left\langle\lambda-\lambda^{\prime}, \xi\right\rangle\right| \leq c\right\} \ll N R^{\epsilon}(1+c) .
$$

Second subset. Once we fix $\lambda$, the lattice points $\lambda^{\prime}$ satisfying

$$
\left|\left\langle\lambda-\lambda^{\prime}, \eta\right\rangle\right| \leq \rho\left|\lambda-\lambda^{\prime}\right|
$$

lie on a spherical segment $\Gamma_{\lambda}$ of opening angle $8 \rho+O\left(\rho^{3}\right)$ and direction $\eta$, or on a spherical cap $\mathcal{T}_{\lambda}$ of radius $\ll \rho R$ and direction $\eta$, on $R \mathcal{S}^{2}$ (see Lemma 3.6). Later we are going to choose $\rho=N^{-8 / 7}$, thus the number of lattice points in $\mathcal{T}_{\lambda}$ of radius $\rho R=o(1)$ is $\ll R^{\epsilon}$. To control the lattice points in each $\Gamma_{\lambda}$, as $\eta$ is of type (ii), we may apply (3.7):

$$
\#\left\{\left(\lambda, \lambda^{\prime}\right):\left|\left\langle\lambda-\lambda^{\prime}, \eta\right\rangle\right| \leq \rho \cdot\left|\lambda-\lambda^{\prime}\right|\right\} \ll N R^{\epsilon}\left(1+R \rho^{1 / 2}\right) .
$$

Third subset. Here we have

$$
\sum \frac{1}{\left\langle\lambda-\lambda^{\prime}, \xi\right\rangle^{2}\left\langle\lambda-\lambda^{\prime}, \eta\right\rangle^{2}}<\frac{1}{c^{2} \rho^{2}} \sum \frac{1}{\left|\lambda-\lambda^{\prime}\right|^{2-\epsilon^{\prime}}} \ll \frac{m^{\epsilon}}{c^{2} \rho^{2}}
$$

via an application of Proposition 3.1. Collecting the estimates (4.13), (4.14), (4.15), and (4.12) we obtain

$$
\mathcal{G} \ll_{\Pi} N R^{\epsilon}(1+c)+N R^{\epsilon}\left(1+R \rho^{1 / 2}\right)+\frac{m^{\epsilon}}{c^{2} \rho^{2}} .
$$

The optimal choice of parameters $(c, \rho)=\left(N^{3 / 7}, N^{-8 / 7}\right)$ yields (4.5) with $a=3 / 7$.

(B) In case $\vec{n}$ is of type (iii), then $\xi$ is of type (ii) and $\eta$ of type (iii). After a relabelling ${ }^{7}, \xi$ is of type (iii) and $\eta$ of type (ii). We modify the proof of part (A) in the following way. In the first subset, by Lemma 3.5 and (3.6),

$$
\#\left\{\left(\lambda, \lambda^{\prime}\right):\left|\left\langle\lambda-\lambda^{\prime}, \xi\right\rangle\right| \leq c\right\} \ll N R^{1 / 2+\epsilon} \cdot\left(R^{1 / 4}+c\right) .
$$

In the second subset, the lattice points in the cap $\mathcal{T}_{\lambda}$ of radius $\ll \rho R$ have the upper bound $R^{\epsilon}\left(1+\rho^{2} R^{3 / 2}\right)$ (Lemma 3.3), while those in each segment $\Gamma_{\lambda}$ are no more than $R^{\epsilon}\left(1+R \rho^{1 / 2}\right)$ (3.7). It follows that

$$
\#\left\{\left(\lambda, \lambda^{\prime}\right):\left|\left\langle\lambda-\lambda^{\prime}, \eta\right\rangle\right| \leq \rho\left|\lambda-\lambda^{\prime}\right|\right\} \quad \ll N R^{\epsilon}\left(1+\rho^{2} R^{3 / 2}\right)+N R^{\epsilon}\left(1+R \rho^{1 / 2}\right) .
$$

Choosing e.g. $(c, \rho)=\left(N^{1 / 14}, N^{-6 / 7}\right)$, we have obtained the bound

$$
\mathcal{G} \ll_{\Pi} N R^{\epsilon}\left(1+\rho^{2} R^{3 / 2}+R \rho^{1 / 2}\right)+N R^{1 / 2+\epsilon}\left(R^{1 / 4}+c\right)+\frac{m^{\epsilon}}{c^{2} \rho^{2}} \ll N^{7 / 4+\epsilon}
$$

proving Lemma 4.4 part (B).

\footnotetext{
${ }^{7}$ Alternatively, one could swap the roles of $\xi, \eta$ when defining the three subsets.
} 


\section{Restriction of Laplace eigenfunctions}

\subsection{Conditional result}

It remains to show Lemma 4.4 part (C). Assuming Conjecture 1.3, one may improve the bound (3.6) for lattice points in spherical segments of given height and larger base radius.

Corollary 4.6 ([28, Corollary 5.6]). Assume Conjecture 1.3. Let $\Gamma \subset R \mathcal{S}^{2}$ be a spherical segment of height $h$ and radius of larger base $k$. Then for every $\epsilon>0$,

$$
\psi \ll R^{\epsilon} \cdot\left(R^{1 / 2}+h\right) .
$$

We introduce the parameters $c=c(N), c^{\prime}=c^{\prime}(N)>0$ and consider the three subsets

- first subset: $\left|\left\langle\lambda-\lambda^{\prime}, \xi\right\rangle\right| \leq c$;

- second subset: $\left|\left\langle\lambda-\lambda^{\prime}, \eta\right\rangle\right| \leq c^{\prime}$;

- third subset: $\left|\left\langle\lambda-\lambda^{\prime}, \xi\right\rangle\right|>c,\left|\left\langle\lambda-\lambda^{\prime}, \eta\right\rangle\right|>c^{\prime}$.

We apply Lemma 4.5 to obtain

$$
\begin{aligned}
\mathcal{G} & \ll_{\Pi} \#\left\{\left(\lambda, \lambda^{\prime}\right):\left|\left\langle\lambda-\lambda^{\prime}, \xi\right\rangle\right| \leq c\right\}+\#\left\{\left(\lambda, \lambda^{\prime}\right):\left|\left\langle\lambda-\lambda^{\prime}, \eta\right\rangle\right| \leq c^{\prime}\right\} \\
& +\sum_{\substack{\left|\left\langle\lambda-\lambda^{\prime}, \xi\right\rangle\right|>c \\
\left|\left\langle\lambda-\lambda^{\prime}, \eta\right\rangle\right|>c^{\prime}}} \frac{1}{\left\langle\lambda-\lambda^{\prime}, \xi\right\rangle^{2}\left\langle\lambda-\lambda^{\prime}, \eta\right\rangle^{2}} .
\end{aligned}
$$

First subset. Once we fix $\lambda$, the lattice points $\lambda^{\prime}$ satisfying

$$
\left|\left\langle\lambda-\lambda^{\prime}, \xi\right\rangle\right| \leq c
$$

lie on a spherical segment $\Gamma_{\lambda}$ of height at most $2 c$ and direction $\xi$ (see Lemma 3.5). By (4.16),

$$
\#\left\{\left(\lambda, \lambda^{\prime}\right):\left|\left\langle\lambda-\lambda^{\prime}, \xi\right\rangle\right| \leq c\right\} \ll N R^{\epsilon}\left(R^{1 / 2}+c\right) .
$$

Second subset. Similarly to the first subset,

$$
\#\left\{\left(\lambda, \lambda^{\prime}\right):\left|\left\langle\lambda-\lambda^{\prime}, \eta\right\rangle\right| \leq c^{\prime}\right\} \ll N R^{\epsilon}\left(R^{1 / 2}+c^{\prime}\right) .
$$

Third subset. Here we simply write

$$
\sum \frac{1}{\left\langle\lambda-\lambda^{\prime}, \xi\right\rangle^{2}\left\langle\lambda-\lambda^{\prime}, \eta\right\rangle^{2}}<\frac{N^{2}}{c^{2} c^{\prime 2}}
$$

Collecting the estimates (4.18), (4.19), (4.20), and (4.17), we obtain

$$
\mathcal{G} \ll_{\Pi} N R^{\epsilon}\left(R^{1 / 2}+c+c^{\prime}\right)+\frac{N^{2}}{c^{2} c^{\prime 2}} \ll N^{3 / 2+\epsilon},
$$

choosing e.g. $c=c^{\prime}=N^{1 / 5}$. This completes the proof of Lemma 4.4 part (C).

\section{A Proofs of auxiliary results}

In this appendix, we prove a couple of auxiliary lemmas.

Proof of Lemma 4.1. We follow [29, §3 and §6] and [28, §3]. Squaring $r_{f}(1.17)$ we obtain

$$
r_{f}^{2}\left((u, v),\left(u^{\prime}, v^{\prime}\right)\right)=\frac{1}{N^{2}} \sum_{\lambda, \lambda^{\prime}} e^{2 \pi i\left\langle\lambda-\lambda^{\prime},\left(u^{\prime}-u\right) \xi+\left(v^{\prime}-v\right) \eta\right\rangle} .
$$


On integrating,

$$
\begin{aligned}
& \iint_{\Pi^{2}} r^{2} d p d p^{\prime}=\iint_{([0, A] \times[0, B])^{2}} \frac{1}{N^{2}} \sum_{\lambda, \lambda^{\prime}} e^{2 \pi i\left\langle\lambda-\lambda^{\prime},\left(u^{\prime}-u\right) \xi+\left(v^{\prime}-v\right) \eta\right\rangle} d u d v d u^{\prime} d v^{\prime} \\
& =\frac{1}{N^{2}} \sum_{\lambda, \lambda^{\prime}} \int_{0}^{A} \int_{0}^{B} e^{2 \pi i\left\langle\lambda-\lambda^{\prime}, u^{\prime} \xi+v^{\prime} \eta\right\rangle} d u^{\prime} d v^{\prime} \int_{0}^{A} \int_{0}^{B} e^{-2 \pi i\left\langle\lambda-\lambda^{\prime}, u \xi+v \eta\right\rangle} d u d v \\
& =\frac{1}{N^{2}} \sum_{\lambda, \lambda^{\prime}}\left|\int_{0}^{A} \int_{0}^{B} e^{2 \pi i\left\langle\lambda-\lambda^{\prime}, u \xi+v \eta\right\rangle} d u d v\right|^{2}=\frac{\mathcal{G}}{N^{2}}
\end{aligned}
$$

To complete the proof of (4.1), by the symmetries it will suffice to show that

$$
\iint_{([0, A] \times[0, B])^{2}}\left[\frac{1}{m}\left(\frac{\partial r_{f}}{\partial u}\right)^{2}+\frac{1}{m^{2}}\left(\frac{\partial^{2} r_{f}}{\partial u \partial u^{\prime}}\right)^{2}\right] d u d v d u^{\prime} d v^{\prime} \ll \frac{\mathcal{G}}{N^{2}}
$$

(see Definition 2.1). One has

$$
\frac{\partial r_{f}}{\partial u}=\frac{2 \pi i}{N} \sum_{\lambda \in \Lambda}\langle\lambda, \xi\rangle e^{2 \pi i\left\langle\lambda,\left(u^{\prime}-u\right) \xi+\left(v^{\prime}-v\right) \eta\right\rangle},
$$

hence, as required in (A.2),

$$
\begin{aligned}
& \iint_{([0, A] \times[0, B])^{2}} \frac{1}{m}\left(\frac{\partial r_{f}}{\partial u}\right)^{2} d u d v d u^{\prime} d v^{\prime} \\
& \ll \iint \frac{1}{N^{2}} \sum_{\lambda, \lambda^{\prime}}\left\langle\frac{\lambda}{|\lambda|}, \xi\right\rangle\left\langle\frac{\lambda^{\prime}}{\left|\lambda^{\prime}\right|}, \xi\right\rangle e^{2 \pi i\left\langle\lambda-\lambda^{\prime},\left(u^{\prime}-u\right) \xi+\left(v^{\prime}-v\right) \eta\right\rangle} d u d v d u^{\prime} d v^{\prime} \\
& \leq \iint \frac{1}{N^{2}} \sum_{\lambda, \lambda^{\prime}} e^{2 \pi i\left\langle\lambda-\lambda^{\prime},\left(u^{\prime}-u\right) \xi+\left(v^{\prime}-v\right) \eta\right\rangle} d u d v d u^{\prime} d v^{\prime}=\frac{\mathcal{G}}{N^{2}}
\end{aligned}
$$

where in the second inequality we applied Cauchy-Schwartz. The calculation for the second derivatives is very similar and we omit it here.

Proof of Lemma 4.5. The upper bound (4.6) is a straightforward application of the triangle inequality. To show (4.7), we integrate and apply the triangle inequality,

$$
\left|\int_{0}^{A} e^{2 \pi i u\left\langle\lambda-\lambda^{\prime}, \xi\right\rangle} d u\right|^{2}=\frac{\left|e^{2 \pi i A\left\langle\lambda-\lambda^{\prime}, \xi\right\rangle}-1\right|^{2}}{4 \pi^{2}\left\langle\lambda-\lambda^{\prime}, \xi\right\rangle^{2}} \leq \frac{1}{\pi^{2}} \cdot \frac{1}{\left\langle\lambda-\lambda^{\prime}, \xi\right\rangle^{2}}
$$

and similarly for the integral over $[0, B]$. This completes the proof of Lemma 4.5.

\section{References}

[1] Jean-Marc Azaïs, José R. León, and Mario Wschebor, Rice formulae and Gaussian waves, Bernoulli 17 (2011), no. 1, 170-193. MR-2797987

[2] Jean-Marc Azaïs and Mario Wschebor, Level sets and extrema of random processes and fields, John Wiley \& Sons, Inc., Hoboken, NJ, 2009. MR-2478201

[3] Dmitry Beliaev and Riccardo W. Maffucci, Coupling of stationary fields with application to arithmetic waves, arXiv preprint arXiv:1912.09470 (2019).

[4] Jacques Benatar and Riccardo W. Maffucci, Random waves on $\mathbb{T}^{3}$ : Nodal area variance and lattice point correlations, International Mathematics Research Notices 2019, no. 10, 3032-3075. MR-3952558

[5] Michael V. Berry, Regular and irregular semiclassical wavefunctions, Journal of Physics A: Mathematical and General 10 (1977), no. 12, 2083. MR-0489542 
[6] Michael V. Berry, Statistics of nodal lines and points in chaotic quantum billiards: perimeter corrections, fluctuations, curvature, Journal of Physics A: Mathematical and General 35 (2002), no. 13, 3025. MR-1913853

[7] Eugene Bogomolny and Charles Schmit, Random wavefunctions and percolation, Journal of Physics A: Mathematical and Theoretical 40 (2007), no. 47, 14033. MR-2438110

[8] Jean Bourgain and Zeév Rudnick, On the nodal sets of toral eigenfunctions, Invent. Math. 185 (2011), no. 1, 199-237. MR-2810800

[9] Jean Bourgain and Zeév Rudnick, Restriction of toral eigenfunctions to hypersurfaces and nodal sets, Geom. Funct. Anal. 22 (2012), no. 4, 878-937. MR-2984120

[10] Jean Bourgain, Zeév Rudnick, and Peter Sarnak, Spatial statistics for lattice points on the sphere I: individual results, Bulletin of the Iranian Mathematical Society 43 (2017), no. 4 (Special Issue), 361-386. MR-3711836

[11] Jean Bourgain, Peter Sarnak, and Zeév Rudnick, Local statistics of lattice points on the sphere, Modern Trends in Constructive Function Theory, Contemp. Math 661 (2012), 269282. MR-3489563

[12] Valentina Cammarota, Nodal area distribution for arithmetic random waves, Transactions of the American Mathematical Society (2019). MR-3988618

[13] Valentina Cammarota, Domenico Marinucci, and Igor Wigman, On the distribution of the critical values of random spherical harmonics, The Journal of Geometric Analysis 26 (2016), no. 4, 3252-3324. MR-3544960

[14] Yaiza Canzani and Boris Hanin, Local universality for zeros and critical points of monochromatic random waves, arXiv preprint arXiv:1610.09438 (2016).

[15] Yaiza Canzani and John A. Toth, Nodal sets of Schrödinger eigenfunctions in forbidden regions, Annales Henri Poincaré 17 (2016), no. 11, 3063-3087. MR-3556516

[16] Shiu-Yuen Cheng, Eigenfunctions and nodal sets, Commentarii Mathematici Helvetici 51 (1976), no. 1, 43-55. MR-0397805

[17] Federico Dalmao, Anne Estrade, and José León, On 3-dimensional Berry's model, arXiv preprint arXiv:1912.09774 (2019).

[18] Manfredo P. do Carmo, Differential geometry of curves and surfaces, Prentice-Hall, Inc., Englewood Cliffs, N.J., 1976, Translated from the Portuguese. MR-0394451

[19] William Duke, Hyperbolic distribution problems and half-integral weight Maass forms, Inventiones mathematicae 92 (1988), no. 1, 73-90. MR-0931205

[20] William Duke and Rainer Schulze-Pillot, Representation of integers by positive ternary quadratic forms and equidistribution of lattice points on ellipsoids, Inventiones Mathematicae 99 (1990), no. 1, 49-57. MR-1029390

[21] Layan El-Hajj and John A. Toth, Intersection bounds for nodal sets of planar Neumann eigenfunctions with interior analytic curves, Journal of Differential Geometry 100 (2015), no. 1, 1-53. MR-3326573

[22] Elena Petrovna Golubeva and Oleg Mstislavovich Fomenko, Asymptotic distribution of integral points on the three-dimensional sphere, Zapiski Nauchnykh Seminarov POMI 160 (1987), 54-71. MR-0906844

[23] Vojtěch Jarník, Über die Gitterpunkte auf konvexen Kurven, Math. Z. 24 (1926), no. 1, 500-518. MR-1544776

[24] Manjunath Krishnapur, Pär Kurlberg, and Igor Wigman, Nodal length fluctuations for arithmetic random waves, Ann. of Math. (2) 177 (2013), no. 2, 699-737. MR-3010810

[25] Thomas Letendre, Expected volume and Euler characteristic of random submanifolds, Journal of Functional Analysis 270 (2016), no. 8, 3047-3110. MR-3470435

[26] Thomas Letendre, Variance of the volume of random real algebraic submanifolds, Transactions of the American Mathematical Society (2017). MR-3917219

[27] Michael Selwyn Longuet-Higgins, The statistical analysis of a random, moving surface, Philosophical Transactions of the Royal Society of London. Series A, Mathematical and Physical Sciences 249 (1957), no. 966, 321-387. MR-0087257 
[28] Riccardo W. Maffucci, Nodal intersections for random waves against a segment on the 3-dimensional torus, Journal of Functional Analysis 272 (2017), no. 12, 5218-5254. MR3639527

[29] Riccardo W. Maffucci, Nodal intersections of random eigenfunctions against a segment on the 2-dimensional torus, Monatshefte für Mathematik 183 (2017), no. 2, 311-328. MR-3641930

[30] Riccardo W. Maffucci, Nodal intersections for arithmetic random waves against a surface, Annales Henri Poincaré 20 (2019), no. 11, 3651-3691. MR-4019200

[31] Domenico Marinucci, Giovanni Peccati, Maurizia Rossi, and Igor Wigman, Non-universality of nodal length distribution for arithmetic random waves, Geometric and Functional Analysis 26 (2016), no. 3, 926-960. MR-3540457

[32] Ivan Nourdin and Giovanni Peccati, Normal approximations with malliavin calculus: from stein's method to universality, vol. 192, Cambridge University Press, 2012. MR-2962301

[33] Ivan Nourdin, Giovanni Peccati, and Maurizia Rossi, Nodal statistics of planar random waves, Communications in Mathematical Physics 369 (2019), no. 1, 99-151. MR-3959555

[34] Ferenc Oravecz, Zeév Rudnick, and Igor Wigman, The Leray measure of nodal sets for random eigenfunctions on the torus, Annales de l'Institut Fourier 58 (2008), no. 1, 299-335. MR-2401223

[35] Stephen O. Rice, Mathematical analysis of random noise, Bell System Technical Journal 23 (1944), no. 3, 282-332. MR-0010932

[36] Maurizia Rossi and Igor Wigman, Asymptotic distribution of nodal intersections for arithmetic random waves, Nonlinearity 31 (2018), no. 10, 4472. MR-3846437

[37] Zeév Rudnick and Igor Wigman, On the volume of nodal sets for eigenfunctions of the Laplacian on the torus, Ann. Henri Poincaré 9 (2008), no. 1, 109-130. MR-2389892

[38] Zeév Rudnick and Igor Wigman, Nodal intersections for random eigenfunctions on the torus, Amer. J. Math. 138 (2016), no. 6, 1605-1644. MR-3595496

[39] Zeév Rudnick, Igor Wigman, and Nadav Yesha, Nodal intersections for random waves on the 3-dimensional torus, Ann. Inst. Fourier (Grenoble) 66 (2016), no. 6, 2455-2484. MR-3580177

[40] Peter Swerling, Statistical properties of the contours of random surfaces, IRE Transactions on Information Theory 8 (1962), no. 4, 315-321. MR-0175653

[41] John A. Toth and Steve Zelditch, Counting nodal lines which touch the boundary of an analytic domain, J. Differential Geom. 81 (2009), no. 3, 649-686. MR-2487604

[42] Igor Wigman, On the distribution of the nodal sets of random spherical harmonics, Journal of mathematical physics 50 (2009), no. 1, 013521 . MR-2492631

[43] Igor Wigman, Fluctuations of the nodal length of random spherical harmonics, Communications in Mathematical Physics 298 (2010), no. 3, 787. MR-2670928

[44] Nadav Yesha, Eigenfunction statistics for a point scatterer on a three-dimensional torus, Annales Henri Poincaré 14 (2013), no. 7, 1801-1836. MR-3115988

Acknowledgments. The author worked on this project mainly during his PhD studies, under the supervision of Igor Wigman. The author is very grateful to Igor for suggesting this very interesting problem, and for insightful remarks. Many thanks to an anonymous referee for helpful corrections. The author was supported by a Graduate Teaching Scholarship, King's College London; by the Engineering \& Physical Sciences Research Council (EPSRC) Fellowship EP/M002896/1 held by Dmitry Belyaev; by Swiss National Science Foundation project 200021_184927. 


\section{Electronic Journal of Probability Electronic Communications in Probability}

\section{Advantages of publishing in EJP-ECP}

- Very high standards

- Free for authors, free for readers

- Quick publication (no backlog)

- Secure publication $\left(\mathrm{LOCKSS}^{1}\right)$

- Easy interface (EJMS²)

\section{Economical model of EJP-ECP}

- Non profit, sponsored by $\mathrm{IMS}^{3}, \mathrm{BS}^{4}$, ProjectEuclid ${ }^{5}$

- Purely electronic

\section{Help keep the journal free and vigorous}

- Donate to the IMS open access fund ${ }^{6}$ (click here to donate!)

- Submit your best articles to EJP-ECP

- Choose EJP-ECP over for-profit journals

\footnotetext{
${ }^{1}$ LOCKSS: Lots of Copies Keep Stuff Safe http://www. lockss.org/

${ }^{2}$ EJMS: Electronic Journal Management System http://www.vtex.lt/en/ejms.html

${ }^{3}$ IMS: Institute of Mathematical Statistics http://www.imstat.org/

${ }^{4}$ BS: Bernoulli Society http://www. bernoulli-society.org/

${ }^{5}$ Project Euclid: https://projecteuclid.org/

${ }^{6}$ IMS Open Access Fund: http://www.imstat.org/publications/open.htm
} 